Article

\title{
A Study on the Braided Fabrication of Fiber Bragg Grating sensor
}

Songbi Lee ${ }^{1}$, Joohyeon Lee ${ }^{1}$ *

${ }^{1}$ Department of Cognitive Science, Yonsei University, 50 Yonsei-ro, Seodaemun-gu, Seoul, Korea 03722 taniasongi@gmail.com (S.L.);ljhyeon@yonsei.ac.kr (J.L.)

*Correspondence: ljhyeon@yonsei.ac.kr (J.L.); Tel.: +82221233108

\begin{abstract}
This study's objective was to propose the use of textile braiding manufacturing methods, thus facilitating the application of the high precision and accurate measurability of optical fiber Bragg grating sensors to various structures. The purpose of this study was to Combine $3 \mathrm{~d}$ braid processing with the optical Bragg grating sensor's accurate metrology. Out of limits of the sensor's epoxy attachment methods, the textile braiding method can make applicable scope diversify. The braiding processing is capable of designing a 3D fabric module processing, multiple objective mechanical fiber arrangement, and material characteristics. Optical stress-strain response conditions were explored through the optimization of design elements between the Bragg grating sensor and braiding. For this study, Bragg grating sensors were located $75 \%$ apart from the fiber center. The sensor core structure is helical of 1.54 pitch. A polyurethane synthetic yarn was braided together with the sensor on the Weaving machine core part in a braiding. Prototyping results, a negative Poisson's ratio makes curled the braided Bragg grating sensor. The number of polyurethane string yarns has been conducted the role of wrap angle in braiding. The 12 strands condition showed an increase in double stress-strain response rate at a Poisson ratio of $1.3 \%$, and 16 strands condition was found to affect the sensor with noise at a Poisson ratio of $1.5 \%$. This study can suggest applying braid processing of the Bragg grating sensor, which is expected to create and develop a new monitoring sensor.
\end{abstract}

Keywords: Bragg grating sensor., auxetic sensor, silica helical core, wrap angle, braid angle

0.

1. Introduction

Fused silica has the characteristics of the high purity of more than $9.999 \%$, excellent heat resistance, thermal stability, chemical stability, excellent light transmittance, and electrical insulation properties. It is resistant to heat and chemical reactions and is useful as an 
optical material for lasers. The development of 3D ultra-fine processing techniques using the diffraction phenomenon of light has been applied as an essential optical element. Precise length measurement experiments have been performed based on the principle of optical interference by previous studies. It can be used as a ruler in the femtosecond laser region, and exact control is possible at $10^{-6}$. Research is needed to easily apply the Femtosecond laser's precision Bragg grating sensor to various applications.

Braid has to go through three other classic manufacturing steps: weaving, knitting, and non-woven. This can make that the braid structure is excellent method in bending strength, impact resistance, torsion efficiency, and energy absorption. Prior studies have reported that composites are in the engineering, aerospace, transportation or medical industries, and various braiding structures and materials development.[1][2][3]

3D module weaving is possible, and a weaving of complex structures is possible, so expansion and contraction of new units is possible through the combination of each module.

By combining the accuracy of the optical Bragg grating sensor with the possibility of $3 \mathrm{~d}$ fabric modular processing in a braiding method, it is possible to measure complicated structures and diversify the sensor application by presenting the possibility of applying the fabrication to the monitoring measurement method.

The objective of this study was to propose the use of woven braiding manufacturing methods, thus facilitating the application of the high precision and accurate measurability of optical fiber Bragg grating sensors to various structures.

\subsection{Fiber Bragg grating sensor}

To design the experiments for this study, Bragg grating sensors were located $75 \%$ apart from the fiber center to increase the response of stress-strain measurements. The Bragg sensor that would be braided was sampled by optimizing it for stress-strain response conditions efficiently to react by the mechanical property of grating core location, core structure (helical core, pitch $1.54 \mathrm{~cm}$ ). The core's frame was designed by permanently modifying a preform level helical (pitch $1.57 \mathrm{~cm}$ ) and applying femtoseconds laser processing for Bragg grating. The size of the processed grid of the silica Bragg grating sensor is $3 \mathrm{~cm}$ and is attached with epoxy to a $15 \mathrm{~cm}$ flexible rod.

\subsection{Principle of Bragg sensor operation}

The light reflected from the end of the optical fiber and the incident light from a standing wave gives a periodic energy change to induce a periodic refractive index change in the optical fiber core to generate a grating. This phenomenon is called light sensitivity. The optical fiber grating device is a photon device that uses light sensitivity in which a 
refractive index changes when a portion of an optical fiber core to which germanium or boron is added is exposed to an ultraviolet laser. The optical fiber grating element can induce periodic refractive index changes in the optical fiber core portion based on light sensitivity, thereby causing changes in light reflection or transmittance [1].

Where $\boldsymbol{n}$ is the effective refractive index of the optical fiber grating, which means the average refractive index when light passes through one period of the Bragg grating, $\Lambda$ means the grating period engraved on the optical fiber. The light of the wavelength of Bragg that satisfies the Bragg condition in the equation is reflected without passing through the Bragg light, and light of other wavelengths passes. The Bragg wavelength reflected from the optical fiber Bragg grating, which can be expressed in the equation, is a function of effective refractive index and grating spacing. When an external physical quantity such as a short-distance strain of the optical fiber Bragg grating is applied, the Bragg wavelength is changed by these values (Equation 1). By accurately measuring the change in the Bragg wavelength, the unknown physical quantity applied to the optical fiber grating can be obtained. The Bragg wavelength is determined by value of the microstructure period and the refractive index nef of the core (Equation 2), [2]. In bending, the stress is determined from the configuration of the fiber. only half the volume of glass is in tension. The stress increases linearly from zero at the neutral axis to a maximum at the surface (Dr. G. Scott Glaesemann, July 2017).

The strain sensitivity of the Bragg grating can be determined by using the Bragg wavelength change. Strain Response $(1-\mathbf{P} e) \boldsymbol{\epsilon}=\Delta \lambda / \lambda[3][4]$.

$$
\begin{array}{r}
(1-\mathbf{P} e) \boldsymbol{\epsilon}=\Delta \lambda / \boldsymbol{\lambda} \text { (Equation 1) } \\
\frac{\Delta \lambda_{B}}{\lambda_{B}}=\frac{\Delta\left(n_{e f} \Lambda\right)}{n_{e f} \Lambda}=\left(1+\frac{1}{n_{e f}} \frac{\partial n_{e f}}{\partial \varepsilon}\right) \Delta \varepsilon=\left(1+p_{e}\right) \Delta \varepsilon=\beta_{\varepsilon} \Delta \varepsilon \text { (Equation 2) }
\end{array}
$$

ße - strain sensitivity of the Bragg grating

pe - photo elastic constant (variation of index of refraction with axial tension)

pe -0.212 (Photo elastic coefficient)

\subsection{Measurement method of Bragg grating sensor}

The interrogator is measured by the amplitude and phase value of the Bragg wavelength's scattered light. According to the photoelastic waveguide response. this deformation applied to the core by an external physical strain [5]. 
At this time, the wavelength variation induced by the change in the spacing of the optical fiber Bragg grating, which is measured by the complex factors of the displacement, curvature along the axis, and the elastic point that can be explained by the laws of physics and the imposed load. A detection method capable of quantitative analysis has a characteristic in which an external force changes the center frequency.

\subsection{Braiding mechanical structure}

While braiding, a bundle of three or more fibers is connected continuously without cutting. All threads are gathered upwards in the center of the track .and then stretched in the vertical direction to form a braid. There are two or more tracks, each with a group of spindles moving in different directions. The angled braid is characterized in that the continuous threads have a structured design element and become a columnar structure. $3 \mathrm{~d}$ weaving of complicated purpose fabrics is possible and has been applied to various industries.[5] Braiding, one of the methods for weaving composite fibers, can be fabricated in the desired direction depending on the strength and stiffness of the desired structure. The braid enables the continuous orientation of the fibers, allowing the mechanical properties' design according to the properties of the material and construction. (G. Guyader et al.,2013) The braiding angles of the yarns in different directions are not identical. The hybrid braiding angles introduce geometrical incompatibility into the longitudinal deformation of the structure, which leads to improved longitudinal stiffness while the bending flexibility is not compromised. The constant and varied braid angles on the conical mandrel's surface determine the strength of the twist, and a particular mechanical property can be designed using an auxiliary structure(Amit Rawal et al.,2015).

\section{Material and method}

\subsection{Design of the braided Sensor}

The core of the sensor used for this study was designed to optimize the blade. This experiment fiber core is located outside at half the fiber volume $35 \mu \mathrm{m}$ from the center of the fiber). It is a helical core with a diameter of 6.3 microns and has a specification with a numerical aperture of 0.21 and a core validity index within $0.05 \%$. It is rotated at high speed in the preforming step and twisted during the process to have a pitch of $15.4 \mathrm{~cm}$ at 50 times per meter and is coated with an acrylate-based UV transparent fiber. The core applied to the sensor is twisted positively at a twist rate of $0.49 \pi$ to form an optical waveguide in the clockwise direction. The fiber is treated with germanium doping, producing a Bragg grating sensor through precision processing using a femtosecond laser process through a phase mask. It has an operating wavelength of $1550 \mathrm{~nm}$, a coating diameter of 185.7 microns, and an optical fiber diameter of 125.6 microns. 
The Bragg grating engraved sensor was attached to the plastic optical fiber with epoxy. When weaving tubular blading, it was fabricated by inserting it together into the bladder core yarn with irradiation of elastic pieces. The outer yarn was made of polypropylene fiber, and the blade angle was woven with a uniform distribution of 45 degrees. The elastic knitting was investigated so that the number of strings for the same yarn of 12 string and 16string could be uniformly distributed to the braided core yarn. The elastic piece irradiation was spirally wound around the tubular bladder core yarn, and an initial angle value of 45 degrees of blading was designated at four uniformly distributed points. All threads were assembled upwards in the center of the orbit and bladed vertically. Three orbits were used, and a blade machine with eight spindle groups moving in different directions was used.

The below figure 1 is an explanatory diagram of the prototype to be used in this experiment. The braiding was composed of polyester nylon. Polyurethane yarn was used in between the braiding yarn and the flexible sensor rod To maintain the uniform internal tension.

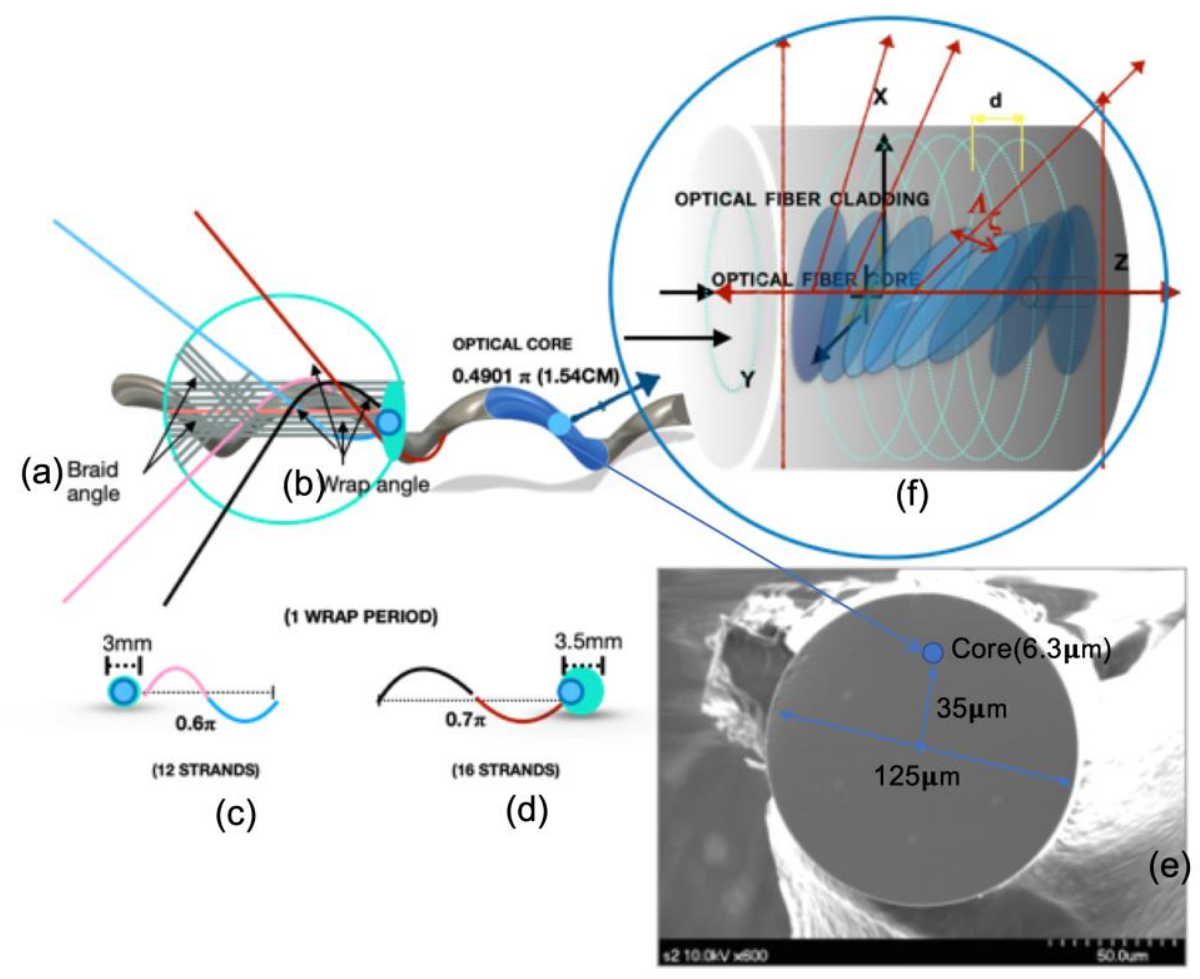

Figure. 1. This figure is for understanding the correlation between the fiber Bragg grating sensor and the braiding angle and wrap angle. figure1(a) is an example of the brazing angle during the braiding process. This figure $1(\mathrm{~b})$ is an example of the polyurethane wrap angle. This figure1(C) shows the wrap pitch $0.6 \pi$ radian and the sensor cross-section diameter of $3 \mathrm{~mm}$ of the 12 -string polyurethane sensor. figure $1(\mathrm{~d})$ shows the 16 -string polyurethane wrap pitch $0.7 \pi$ radian and the sensor cross-section diameter of $3.5 \mathrm{~mm}$. figure $1(\mathrm{C})$ and (d) are samples after braiding, and figure $1(\mathrm{e})$ are electron micrographs of the braided sensor. this figure1(e) shows a core position in the optical fiber 
of $125 \mu \mathrm{m}$ diameters. And figure1(e)shows the Bragg grating sensor core of $6.3 \mu \mathrm{m}$ diameters. This figure1(f) is aimed to understand the process of stress-strain response applied to the Bragg grating sensor by the braiding process.

\subsection{Methodology}

A comparative analysis was conducted on the sensor's signal responsivity with the epoxy attached at the flexible rod sensor and undergone a braiding sensor. The experimental results were recorded. The braiding conditions were analyzed by the size of the sensor signal, and the morphology changes' reliability. The valid responses of the sensor in the context of the conditions of the braiding and wrap investigations were analyzed based on the changes in internal shear stress to calculate the range of investigated braid state.

\subsection{Negative Poisson's ratio}

Poisson's ratio is one of the characteristics of a material. When a force acts on a material, the material's deformation occurs in the direction in which it is applied. If the tensile force acts on the material, it is stretched in the direction of the tensile force. The Poisson's ratio represents the ratio between the horizontal strain and the vertical strain (Equation 3). In other words, the anxiety decreases for the direction of a move of the load and increases the vertical direction and has a positive Poisson's ratio. In Figure 2 below, the principle of Poisson's ratio was explained by connecting the angle and pitch of the wrap angle with tubular braiding. In this experiment, two braided angles and wrap angles are applied to explore the structural principle that negatively affects Poisson's ratio. It is a common phenomenon that the material tends to become thinner when stretched. Rarely, some materials contract or stretch when stretched horizontally, indicating a negative value of the Poisson's ratio (PR). PR is called' auxetics. The negative Poisson's ratio enables the stiffness and resistance to be actively increased to respond to external stress action loads [6].

$$
\nu=\left|\frac{\text { Lateral strain }}{\text { Axial strain }}\right|=-\frac{\epsilon_{y}}{\epsilon_{x}}=-\frac{\epsilon_{z}}{\epsilon_{x}}
$$

(Equation 3) 


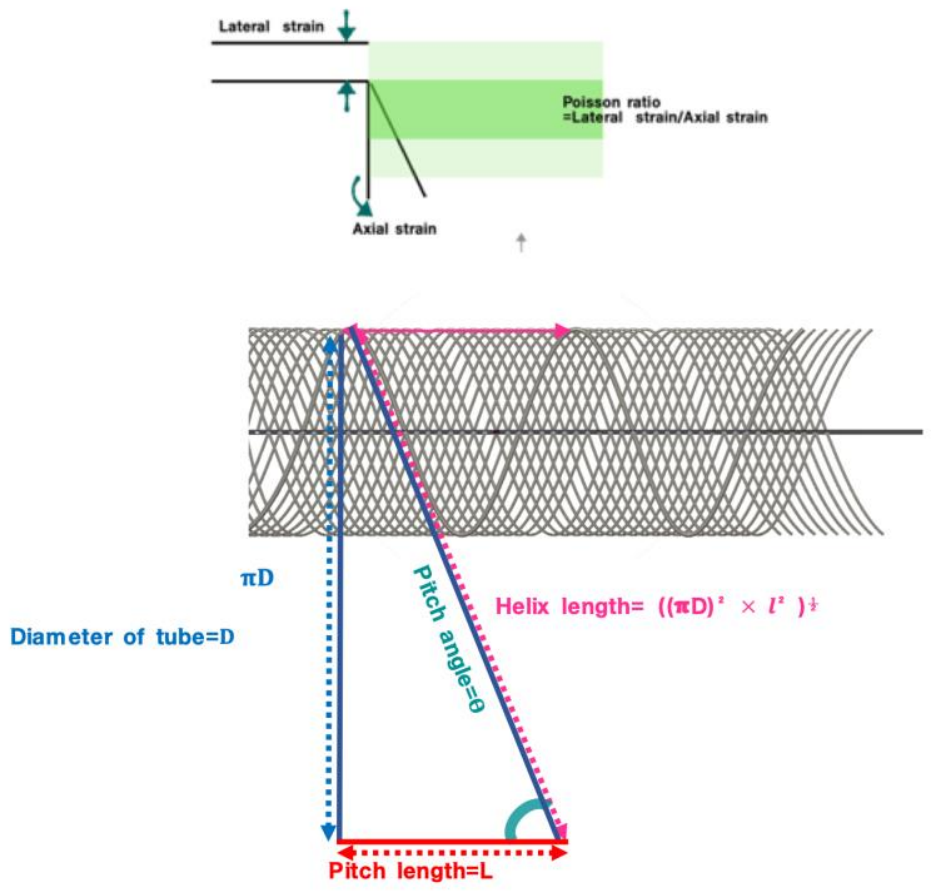

Figure. 2. figure2(a) is a drawing to understand the definition of Poisson's ratio. Figure 2(b) shows the lap angle, lap yarn pitch, and the circumferential length of the braided pipe cross-section, causing a negative Poisson's ratio in tubular braiding.

\subsubsection{Measurement of Bragg Wavelength Variation of Interrogator}

The interrogator is measured by the amplitude and phase value of the Bragg wavelength's scattered light. According to the waveguide, a photoelastic response by deformation applied to the core by an external physical strain. Wavelength shift induced by the change in the spacing of the optical fiber Bragg grating is measured by the complex factors of the displacement, the curvature along the axis, and the elastic point that can be explained by the laws of physics and the imposed load. How to measure It calculates all distributions of reflected light between the start point and endpoint of the sensor peak over the threshold light amount to find the distribution center and includes specifications for the calculation method suitable for the change in the reflected light amount distribution. It is measured by calculating $\Delta \mathrm{N}$ and the amount of change in the fractional part, ${ }^{\Delta} \varepsilon[7]$. 


\subsection{Experiment}

\subsubsection{Subject}

The sensor unit length $(1 \mathrm{~cm})$ and angle (1 degree) were measured before and after the blading. And the maximum and minimum values of the stress-strain response were investigated.

\subsubsection{Experimental device and tools}

For the protocol, the sensor strain measurement behavior was controlled using a device having an angle, and length adjustment range used for stage setup was used. It can be adjusted manually and has a $1 \mathrm{~mm}$ and 1 -degree adjustment resolution. The metronome controlled the speed of the experiment. $10 \mathrm{kHz}$ measurable interrogator figure 3 $((a) c)$ was used to enable real-time monitoring measurements.

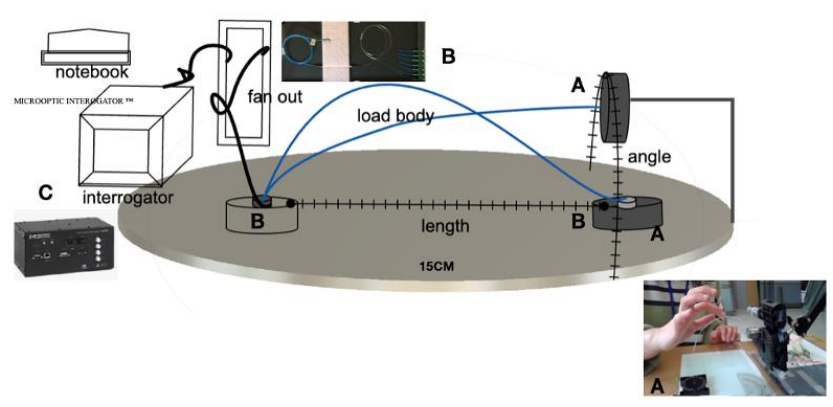

(a)

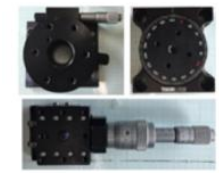

(c)

Figure. 3. this shows the measurement equipment used in the experiment. Figure 3 B is a fanout that can accurately connect the laser and Bragg wavelength shift to the interrogator with a Bragg grating core figure $\left.3 \mathrm{~A}_{\mathrm{r}}(\mathrm{c})\right)$ is a passive optical machine with a $1 \mathrm{~mm}$ resolution. Figure $3 \mathrm{C}$ is the 10 $\mathrm{kHz}$ interrogator, and Figure $3(\mathrm{~b})$ is the experimental picture.

\subsubsection{Protocol}

The stress-strain response was carried out through repeated experiments of increasing and decreasing the unit length and angle. Also, the maximum and minimum val- 
ues of the stress-strain response were investigated. The reliability coefficient verified sensor reliability (internal consistency) in the repeated experiment. The experimental limiting condition is to use an axial fixer at both ends of the prototype to ensure that the optical fiber axis is fixed. The length of the moving line and the optical waveguide axis move on one plane. Three sets of experiments were performed at metronome 60 at a regular interval of $1 \mathrm{~cm}$ and a 10-degree. According to the repetitive motion, the reliability of the test was presented to the reliability coefficient.

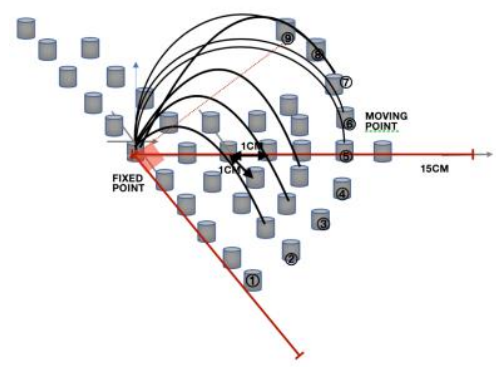

(a) length,angle protocol

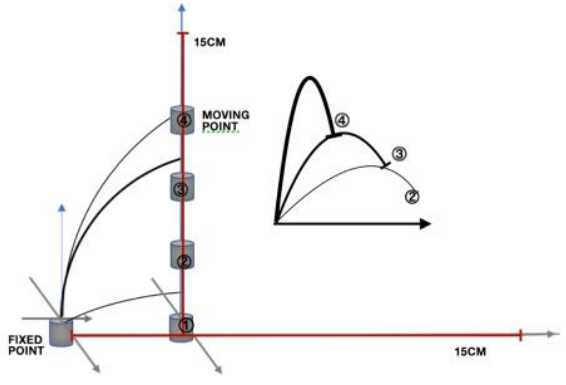

(b) maximum and minimum values protocol

Figure. 4. Figure 4(a) is a diagram of an experimental measurement device with a length of 1 $\mathrm{cm}$ and an angle of 10 degrees. Figure4(B) is an experimental measurement device diagram for measuring the maximum and minimum values. Each fixed position and moving position are selected, and rubber tabs for fixed the optical axis are installed at both ends of the sensor.

\subsection{4 prototype}

The prototype was produced with three types, 12 strands braided sensor, 16 strands braided sensor, and flexible load sensor before braid. As a result of prototyping, 12 strands braided sensor induced cyclic curling with a diameter of $6 \mathrm{~cm}$, and 16 strands braided sensor were produced with cyclic curling with a diameter of $3 \mathrm{~cm}$.

Table. 1. Table 1 shows the state of the sensor before and after the braid. Table 1(c),(d) shows a case where 12 polyurethanes are braided, and 16 polyurethanes are braided. The diameter of the cross-section of the sensor and the diameter of the helical size is indicated.

\begin{tabular}{cccc}
\hline parameter & $\begin{array}{c}\text { flexible rod } \\
\text { frame }\end{array}$ & 12 strands & 16 strands \\
\hline Cross section Diameter (cm) & 0.2 & 0.3 & 0.35 \\
Initial braid angle (degree) & NO & 45 & 45 \\
Number of polyurethane string (N) & NO & 12 & 16 \\
Braided sensor body curling Diameter(cm) & 9 & 6 & 3 \\
\hline
\end{tabular}


(a) Before braid

(b) After braid

(c) 12 strands (d) 16 strands

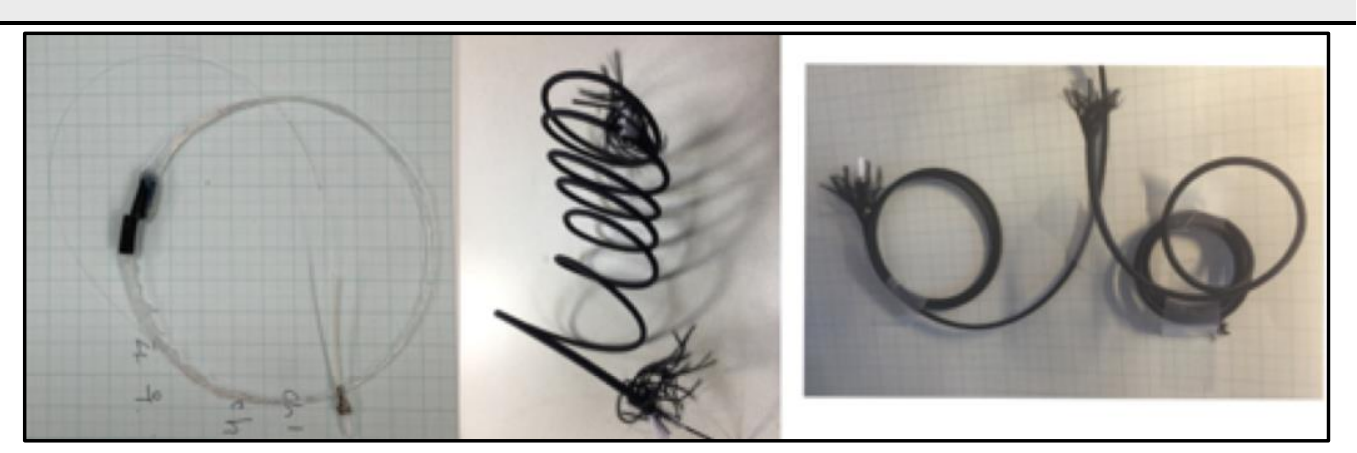

\section{Result}

The experimental results showed a positive braiding range and interconnected relationship by the stress-strain response to the fast waveguide axis strain. These results were used to propose the application standards for sensor braiding. String cords were made to have helical curling by using rubber elasticity. Under Figure $5(A, B, C)$ the same braid conditions, the number of polyurethane yarns caused a change in internal stress and a negative Poisson's ratio. As a result, 16 strands sensor was made with a curling diameter of $3 \mathrm{~cm}$, and 12 strands sensor was made with a curling diameter of $6.6 \mathrm{~cm}$. The 16 strands sensor make pre-strains, and I observed noise on the morphology. The 16 strands sensor was investigated for non-conformance conditions. It is presented in Figure 5.

12 strands sensor was observed to have maximum and minimum wavelength shifts with the same morphology of flexible rod sensor condition so that 12 strands sensor testing be continued with a flexible rod sensor. The result was analyzed using wavelength shift rates before and after braiding. Post-braiding, the loading body frame sensory strain response rate decreased by 0.4 times in 12 strands sensor, while the angle also reduced by 0.3 times. The maximum and minimum values were the same before and after braiding, but the sensor reactivity (wavelength shift speed) increased twofold. 


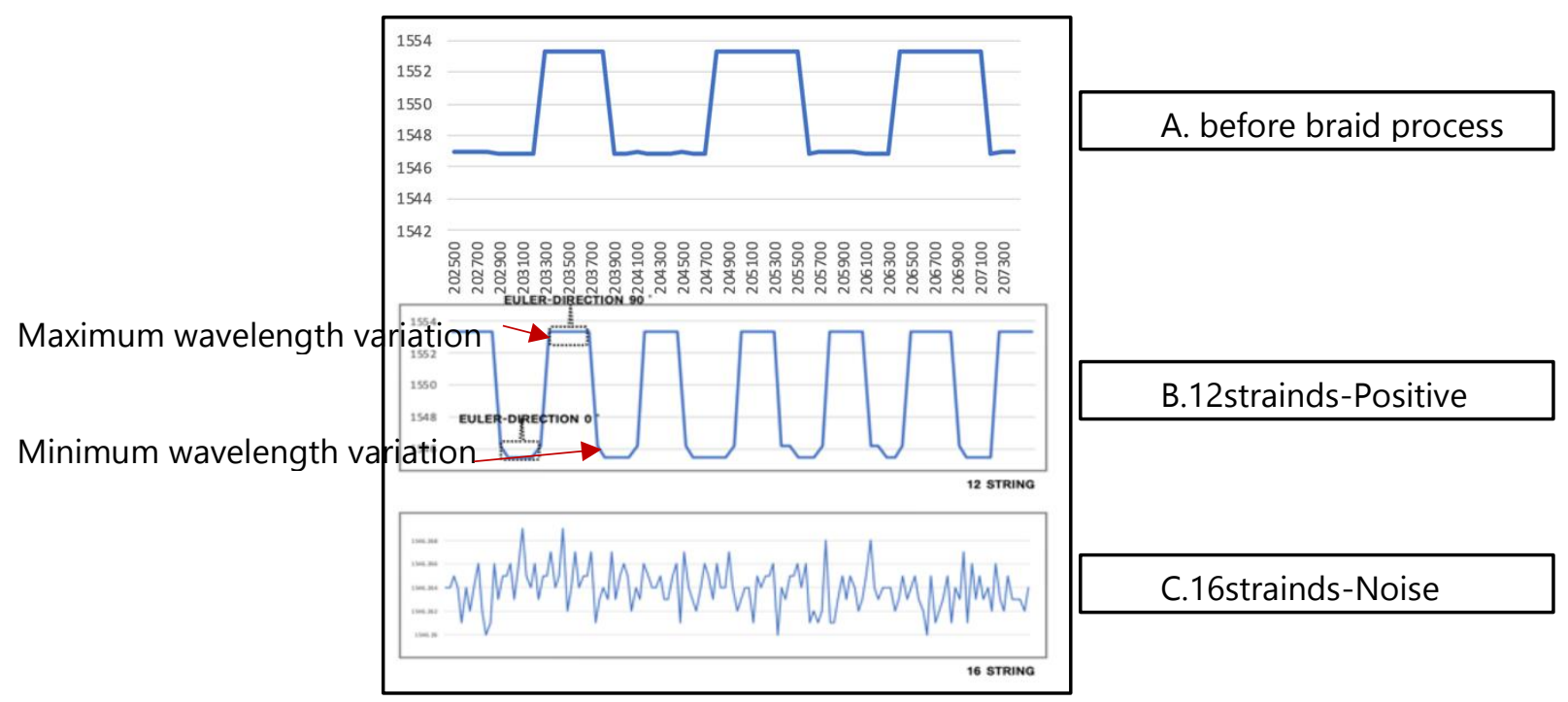

Figure. 5. In the experiment of Figure 5, the wavelength variation for the change of the maximum and minimum values was measured. Figure 5 shows the morphology of before figure $5(A)$ and after figure5((B), (C)) braid. Before the braid figure5(A) and after the 12 strands condition's braid figure 5 (B) showed the effectiveness of the sensor, but the 16 strands condition's braid figure 5(C) was observed in noise signal.

a. Before braid

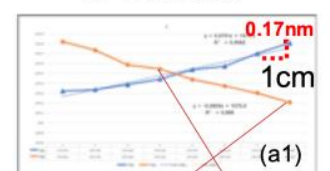

(a1)
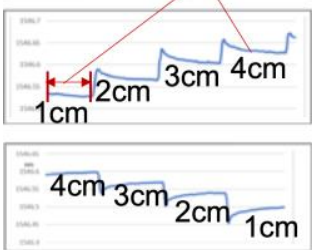

(a2) b. After braid(12 strands)

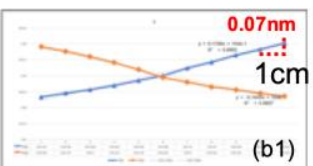

(b1)

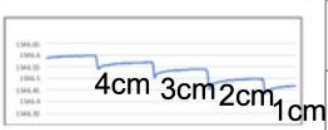

$1 \mathrm{~cm} 2 \mathrm{~cm}^{3 \mathrm{~cm}} 4 \mathrm{~cm}$

(b2)

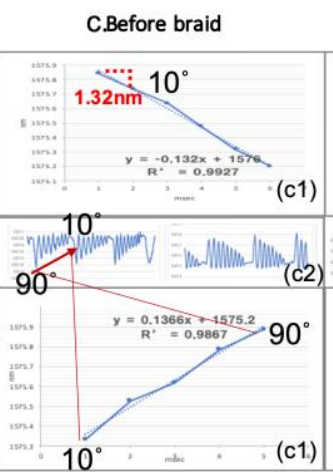

(c1) d. After braid(12 strands)

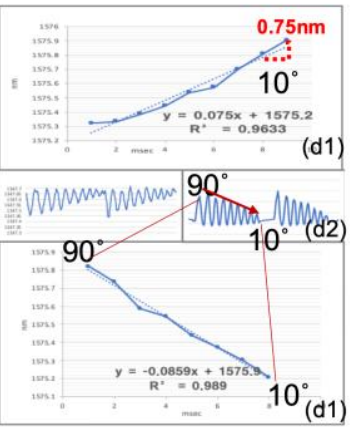

Figure. 6. Figure6 shows the morphology of comparing the sensor's length and angle unit wavelength variations and the maximum and minimum values of the sensors before and after braiding under the same conditions. Figure6((a1) (b1) (c1) (d1)) suggests the linearity of wavelength variation through the trend line. 
Table. 2. Table2 showed 3 prototype investigation data in this study (length, angle maximum and minimum values, Confidence constant-Cronbach's alpha)

\begin{tabular}{|c|c|c|c|c|c|}
\hline subject & \multicolumn{5}{|c|}{ length (wavelength shift/1cm ) } \\
\hline \multirow{6}{*}{ flexible frame } & $1 \mathrm{~cm}$ & $2 \mathrm{~cm}$ & $3 \mathrm{~cm}$ & $4 \mathrm{~cm}$ & $5 \mathrm{~cm}$ \\
\hline & $6 \mathrm{~cm}$ & $7 \mathrm{~cm}$ & $8 \mathrm{~cm}$ & $9 \mathrm{~cm}$ & $10 \mathrm{~cm}$ \\
\hline & $1547.337 \mathrm{~nm}$ & $1547.409 \mathrm{~nm}$ & $1547.44 \mathrm{~nm}$ & $1547.519 \mathrm{~nm}$ & $1547.53 \mathrm{~nm}$ \\
\hline & $1547.563 \mathrm{~nm}$ & $1547.568 \mathrm{~nm}$ & $1547.594 \mathrm{~nm}$ & $1547.619 \mathrm{~nm}$ & $1547.63 \mathrm{~nm}$ \\
\hline & \multicolumn{5}{|c|}{$\begin{array}{l}y=0.172 x+1575.2 \\
=0.9562 \quad(1 s t, 2 s t, 3 s t \text { mean })\end{array}$} \\
\hline & \multicolumn{5}{|c|}{ Standardized Cronbach's alpha. } \\
\hline \multirow{4}{*}{$\begin{array}{c}12 \text { strands } \\
\text { braid }\end{array}$} & $1575.321 \mathrm{~nm}$ & $1575.392 \mathrm{~nm}$ & $1575.465 \mathrm{~nm}$ & $1575.52 \mathrm{~nm}$ & $1575.355 \mathrm{~nm}$ \\
\hline & $1575.63 \mathrm{~nm}$ & $1575.71 \mathrm{~nm}$ & $1575.79 \mathrm{~nm}$ & $1575.86 \mathrm{~nm}$ & $1575.948 \mathrm{~nm}$ \\
\hline & \multicolumn{5}{|c|}{$\begin{array}{c}y=0.0701 x+1575.2 \\
R^{2}=0.9562 \quad(1 s t, 2 s t, 3 s t \text { mean })\end{array}$} \\
\hline & \multicolumn{5}{|c|}{$\begin{array}{c}\text { Standardized Cronbach's alpha. } 0.861 \text { (3st) } \\
\text { ( } \boldsymbol{\alpha} \geqq 0.9 \text { (excellent), } 0.8 \leqq \boldsymbol{\alpha} \leqq 0.9 \text { (qood), } 0.7 \leqq \boldsymbol{\alpha} \leqq 0.8 \text { (acceptable)) }\end{array}$} \\
\hline \multirow{2}{*}{$\begin{array}{c}16 \text { strands } \\
\text { braid }\end{array}$} & \multicolumn{5}{|c|}{ NO } \\
\hline & & angle( & lavelength shift & 1degree) & \\
\hline \multirow[t]{7}{*}{ flexible frame } & $0^{\circ}$ & $10^{\circ}$ & $20^{\circ}$ & $30^{\circ}$ & $40^{\circ}$ \\
\hline & $50^{\circ}$ & $60^{\circ}$ & $70^{\circ}$ & $80^{\circ}$ & $90^{\circ}$ \\
\hline & $1547.7 \mathrm{~nm}$ & $1547.671 \mathrm{~nm}$ & $1547.611 \mathrm{~nm}$ & $1547.597 \mathrm{~nm}$ & $1547.571 \mathrm{~nm}$ \\
\hline & $1547.561 \mathrm{~nm}$ & $1547.538 \mathrm{~nm}$ & $1547.527 \mathrm{~nm}$ & $1547.496 \mathrm{~nm}$ & $1547.458 \mathrm{~nm}$ \\
\hline & \multicolumn{5}{|c|}{$y=0.134 x+1547.4$} \\
\hline & \multicolumn{5}{|c|}{$\mathrm{R}^{2}=0.9827 \quad(1 \mathrm{st}, 2 \mathrm{st}, 3 \mathrm{st}$ mean $)$} \\
\hline & \multicolumn{5}{|c|}{$\begin{array}{c}\boldsymbol{\alpha} \geqq 0.9 \text { (excellent), } 0.8 \leqq \boldsymbol{\alpha} \leqq 0.9 \text { (good), } 0.7 \leqq \boldsymbol{\alpha} \leqq 0.8 \text { (acceptable) } \\
0.6 \leqq \boldsymbol{\alpha} \leqq 0.7 \text { (questionable), } 0.5 \leqq \boldsymbol{\alpha} \leqq 0.6 \text { (poor), } \boldsymbol{\alpha} \leqq 0.5 \text { (unacceptable) }\end{array}$} \\
\hline \multirow{3}{*}{$\begin{array}{c}12 \text { strands } \\
\text { braid }\end{array}$} & $1547.453 \mathrm{~nm}$ & $1547.467 \mathrm{~nm}$ & $1547.493 \mathrm{~nm}$ & $1547.512 \mathrm{~nm}$ & $1547.563 \mathrm{~nm}$ \\
\hline & $1547.568 \mathrm{~nm}$ & $1547.594 \mathrm{~nm}$ & $1547.612 \mathrm{~nm}$ & $1547.63 \mathrm{~nm}$ & $1547.451 \mathrm{~nm}$ \\
\hline & \multicolumn{5}{|c|}{$y=0.0233 x+1547.4$} \\
\hline
\end{tabular}




\begin{tabular}{|c|c|c|}
\hline & \multicolumn{2}{|c|}{$\mathrm{R}^{2}=0.9827(1 \mathrm{st}, 2 \mathrm{st}, 3 \mathrm{st}$ mean $)$} \\
\hline & $\begin{array}{r}\text { Standardize } \\
\boldsymbol{\alpha} \geqq 0.9 \text { (excellent), } 0 \\
0.6 \leqq \boldsymbol{\alpha} \leqq 0.7 \text { (questiona }\end{array}$ & $\begin{array}{l}\text { nbach's alpha. } 0.884 \text { (3st) } \\
0.9 \text { (good), } 0.7 \leqq \boldsymbol{\alpha} \leqq 0.8 \text { (acceptable) } \\
5 \leqq \boldsymbol{\alpha} \leqq 0.6 \text { (poor), } \boldsymbol{\alpha} \leqq 0.5 \text { (unacceptable) }\end{array}$ \\
\hline \multirow[t]{2}{*}{$\begin{array}{l}16 \text { strands } \\
\text { braid }\end{array}$} & & NO \\
\hline & \multicolumn{2}{|c|}{ maximum and minimum values of the stress strain response } \\
\hline flexible frame & $\begin{array}{cc}0^{\circ} & 90^{\circ} \\
1546.901 \mathrm{~nm} & 1549.925 \mathrm{~nm}\end{array}$ & $\begin{array}{c}y=-3.567 x+1554.1 \\
R^{2}=1 \\
(1 s t, 2 s t, 3 s t \text { mean })\end{array}$ \\
\hline $\begin{array}{l}12 \text { strands } \\
\text { braid }\end{array}$ & $1546.916 \mathrm{~nm} \quad 1549.483 \mathrm{~nm}$ & $\begin{array}{c}y=-6.424 x+1559.7 \\
R^{2}=1 \\
(1 s t, 2 s t, 3 s t \text { mean })\end{array}$ \\
\hline $\begin{array}{c}16 \text { strands } \\
\text { braid }\end{array}$ & \multicolumn{2}{|c|}{ NO } \\
\hline
\end{tabular}

The decrease in stress-strain response of angle and length displacement can be interpreted to be acted Pressure to free movement. However, in the internal polyurethane 12 strands condition, the sensor reactivity increased twofold due to internal helical auxetic stress. 


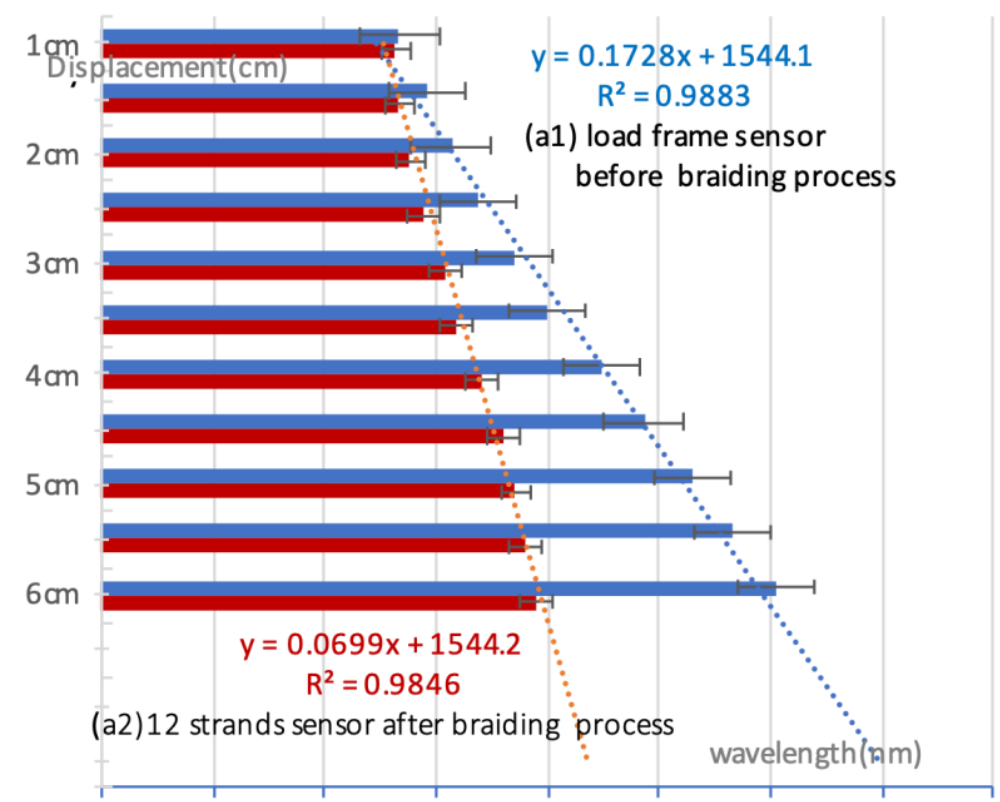

1,543. 0 1,543. 5 1,544. 0 1,544.5 1,545.0 1,545.5 1,546.0 1,546.5 1,547.0

Figure. 7. Figure7 is the result of Figure 6. The reliability value of each experiment was presented by the reliability constant for each result value. Figure 7 shows the stress-strain response change before and after braid. figure7(a1) show wavelength shift for $1 \mathrm{~cm}$ displacement before braid. Figure7(a2) show wavelength shift for $1 \mathrm{~cm}$ displacement After braid.
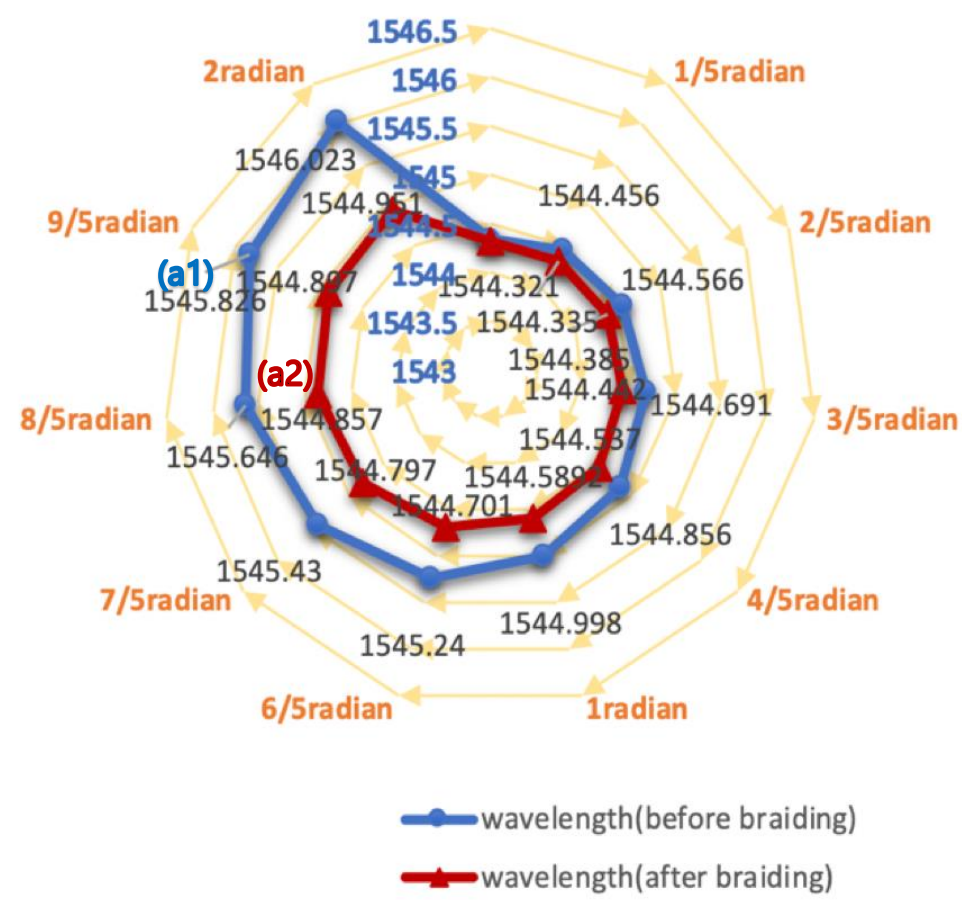
Figure. 8. figure8 (a2) is the wavelength variation after braid. this shows the wavelength shift according to 1 radian, figure $8(\mathrm{a} 1)$ shows the sensor stress-strain wavelength shift before braiding.

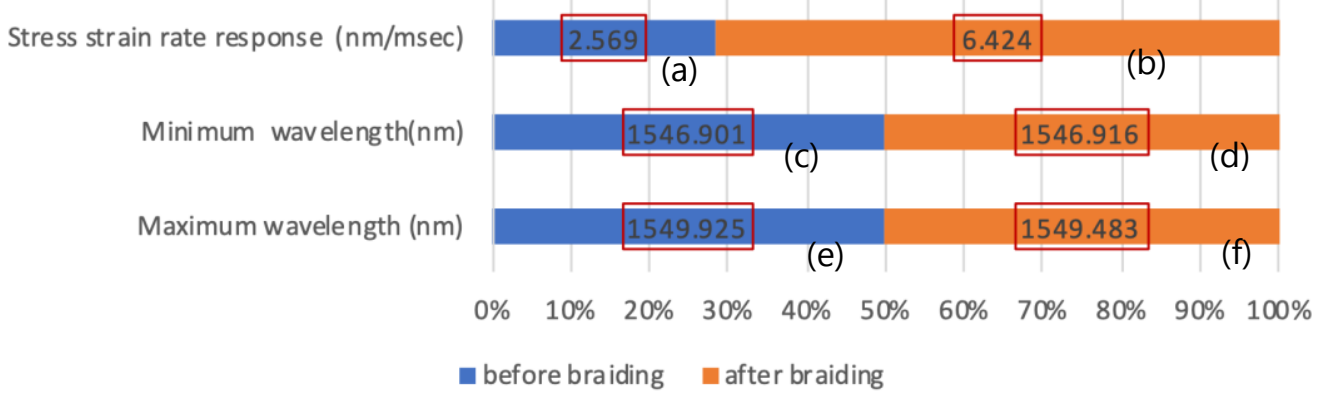

Figure. 9. Figure9 compares the stress-strain response to the optical waveguide axis before and after the braid. Figure $9((a),(b))$ shows the fast rate of responsiveness to stress-strain. Figure $9((c)(d))$ shows the stress-strain response minimum (0\%) before and after braid on the optical waveguide axis. Before and after the stress-strain response, maximums (100\%) were compared in figure $9((c)$, (e)).

This was due to the wrap yarn role of polyurethane, which resulted in positive interaction and fast stress-strain response from the helical auxetic stress in the Bragg sensor core. It is presented in Figure 9. The range of the helical auxetic stress was calculated using the conditions for 12 and 16 strands sensor. The impact range of the helical auxetic distortion rate in the sensors was analyzed in connection to the fiber optic sensor's cores pitch cycles in figure9. 


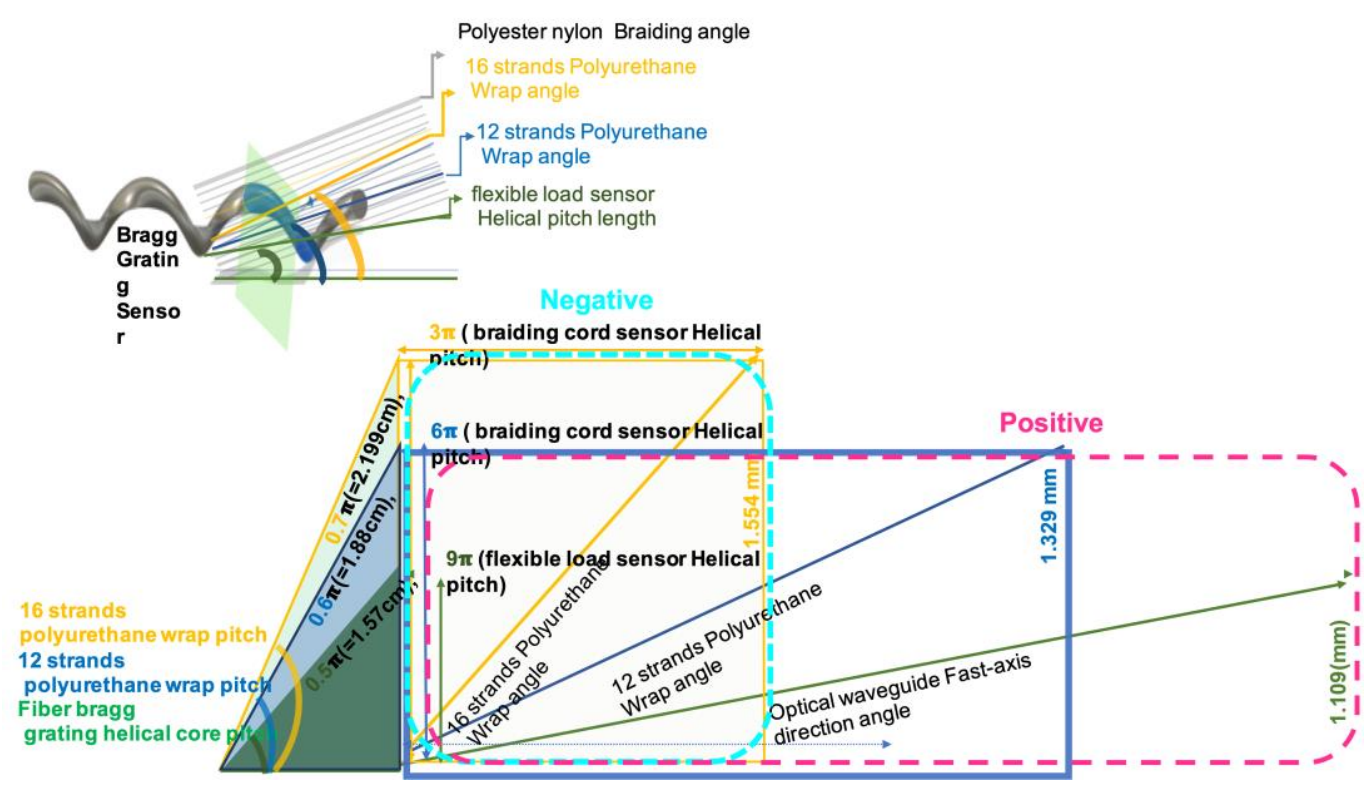

Figure. 10. Figure10 summarizes the experimental results and the conditions related to the sensor and the noise range according to braid condition.

The fiber optic cores have a cycle of $1.54 \mathrm{~cm}$ with a period of $0.49 \pi$. From $0.5 \pi$ to 0.6 $\pi, 6 \mathrm{~cm}$ torsion causes the internal tension on the Bragg grating in the sensors to increase twofold, thus increasing the reactivity by twice as much as before braiding. However, 16 strands sensor have $0.7 \pi$ Helical structures with an internal diameter of $3 \mathrm{~cm}$, which exceeds inner tension in the sensors and results in noise being measured.12 strands sensor with a helical diameter of $6 \mathrm{~cm}$ and the sensor Optical waveguide's vertical section of $3 \mathrm{~mm}$ were used. In figure 10, 16 strands sensor with a helical diameter of $3 \mathrm{~cm}$ and Optical waveguide's vertical section $35 \mathrm{~mm}$ were used. Each measured value was recorded in the experimental figure 10 .

\section{Discussion}

The range of the helical auxetic stress was calculated using the conditions for the 12 and 16- strands sensor. The impact range of the helical auxetic distortion rate in the sensors was analyzed in connection to the cycles of the fiber optic sensor's cores pitch. The Poisson ratio can interpret the tension range impacting the fiber optic cores.

Normal tension (Figure 11) represented the strain placed on the internal Bragg grating based on the same modification rate of $1.1 \%$. The sensors' inner tension was calculated within the range (1.3\%) that would produce a mutually beneficial relationship at the same ratio of increase as the critical 
point tolerance range. It is presented in the positive auxetic tension of Figure 11. Negative auxetic pressure is a diagram that outlines Bragg grating conditions when it increases to the point outside of the critical limit (1.6\%) as a result of internal compression.

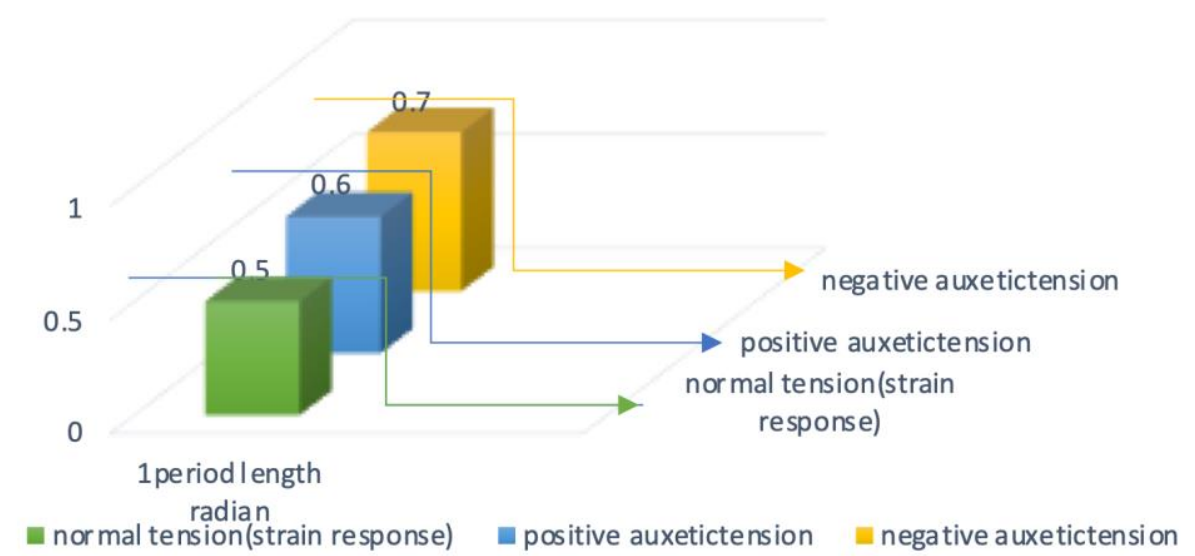

Figure. 11. Figure11 shows the braid effective range of the sensor.

\section{Conclusion}

Using a braid weaving machine, it braided at the core Loom center with the sensor and polyurethane composite yarn. The braided sensor was curled as a result of a negative Poisson's ratio. The mutually beneficial relationship range and noise range of the sensor were differentiated based on the number of braided polyurethane strands. For 12 strands sensor, a Poisson's ratio of $1.3 \%$ resulted in a twofold stress-strain reactivity increase. For 16 strands sensor, a 1.5\% Poisson's ratio resulted in pre-strain noise. These were analyzed as braiding tensor factors that impacted the core, and they can be described in three different levels. Level 1 was defined as external stress-strain factors that occurred only until the point of cladding. At this level, there was no tensor identified the core that resulted in wavelength shifts in the Bragg grating. Level 2 was defined as fiber optic stress-strain factors that occurred up to the outer levels of the core's surface. At this level, tensors in 
the core that resulted in wavelength shifts were determined to be horizontal factors. Level 3 was defined as the strain that occurred within the internal core to $50 \%$ of the center $n$ the same direction as the light wave axis. At this level, tensors in the core resulted in wavelength shifts that were determined to be both horizontal and vertical factors. In terms of conditions for braiding conditions, the requirements for a mutually beneficial relationship in the sensors were explored. As a result, the conditions for mutually beneficial relationships were confirmed to be a Poisson's ratio of $1.3 \%$ at Level 3. 3D fabrication modules due to braiding many present expectations for the application of sensors in complicated and convoluted areas of design. 
Appendix A. Prototype (12 strands braid type) confidence constant investigation data)

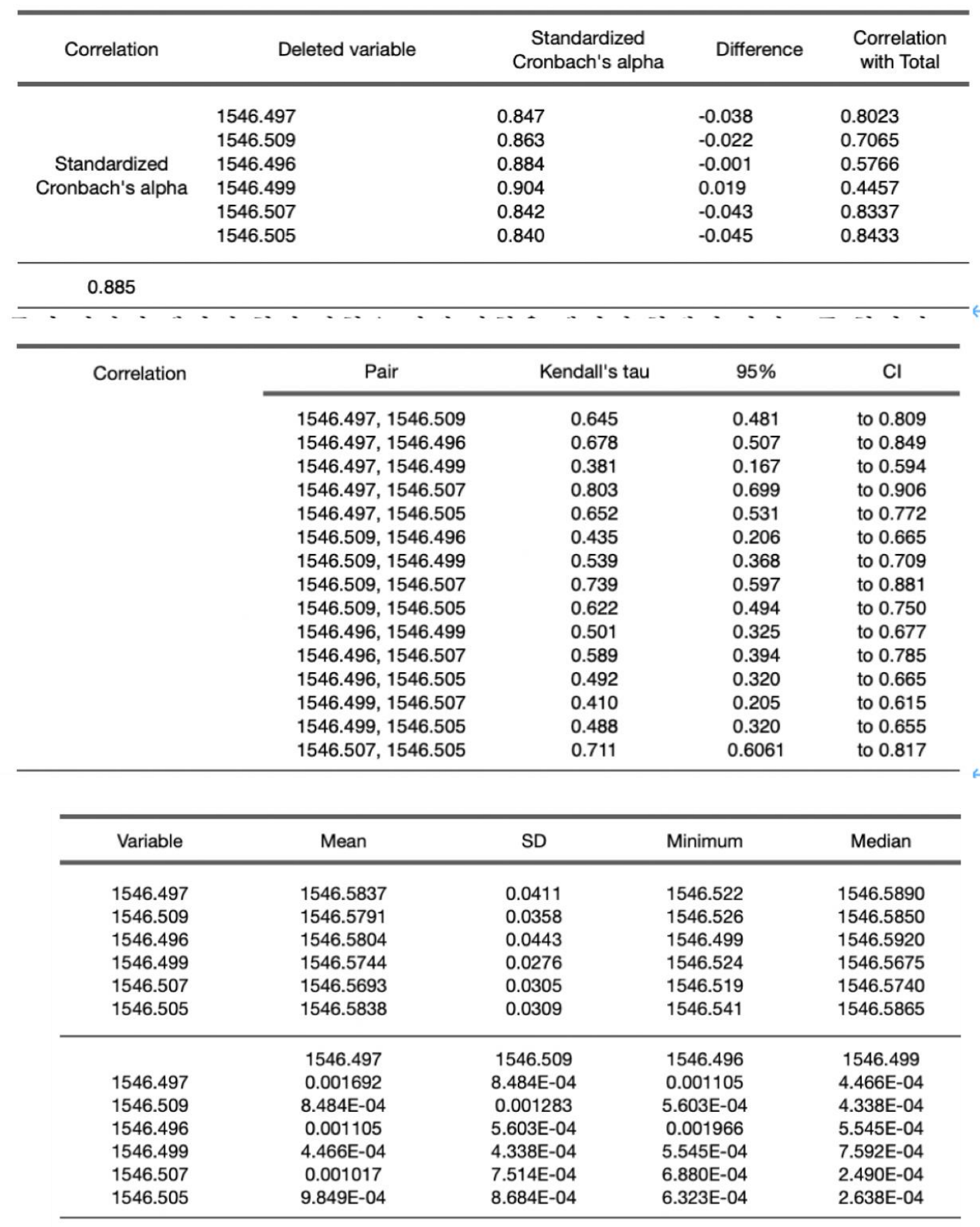

\section{References}

[1].Ahmadi M, et al. An experimental study on mechanical properties of GFRP braid-pultruded composite rods. EXPRESS Polym Lett 2009; 3(9): 560-5688. 
[2].Rana, S., Zdraveva, E., Pereira, C., Fangueiro, R., \& Correia, A. G. (2014). Development of hybrid braided composite rods for reinforcement and health monitoring of structures. ScientificWorldJournal, 2014, 170187. doi:10.1155/2014/170187

[3].Zou, Q., Xue, W., Lin, J., Fu, Y., Guan, G., Wang, F., \& Wang, L. (2016). Mechanical characteristics of novel polyester/NiTi wires braided composite stent for the medical application. Results in Physics, 6, 440-446. doi:10.1016/j.rinp.2016.07.007

[4]Zou, Q., Xue, W., Lin, J., Fu, Y., Guan, G., Wang, F., \& Wang, L. (2016). Mechanical characteristics of novel polyester/NiTi wires braided composite stent for the medical application. Results in Physics, 6 , 440-446. doi:10.1016/j.rinp.2016.07.007

[5]Amit Rawal, Srijan Gupta, Harshvardhan Saraswat and Apurv SibalGeometrical modeling of nearnet shape braided preformsTextile Research Journal,2015, Vol. 85(10) 1055-1064

[1]Ahmadi, M. S., Johari, M. S., Sadighi, M., \& Esfandeh, M. (2009). An experimental study on mechanical properties of GFRP braid-pultruded composite rods. Express Polymer Letters, 3(9), 560-568. doi:10.3144/expresspolymlett.2009.70

[2]Campanella, C. E., Cuccovillo, A., Campanella, C., Yurt, A., \& Passaro, V. M. N. (2018). Fibre Bragg Grating Based Strain Sensors: Review of Technology and Applications. Sensors (Basel), 18(9). doi:10.3390/s18093115

[3]Chen, J., Xu, Q., Xia, Z., \& Xu, W. (2012). An experimental study of influence of filament and roving location on yarn properties during embeddable and locatable spinning. Fibers and Polymers, 13(9), 1196-1200. doi:10.1007/s12221-012-1196-3

[4]Chen, X., Du, F., Guo, T., Lao, J., Zhang, X., Zhang, Z., . . Guan, B.-O. (2017). Liquid Crystal-Embedded Tilted Fiber Grating Electric Field Intensity Sensor. Journal of Lightwave Technology, 35(16), 3347-3353. doi:10.1109/jlt.2016.2643163

[5]Durana, G., Kirchhof, M., Luber, M., de Ocariz, I. S. Á., Poisel, H., Zubia, J., \& Vazquez, C. (2009). Use of a Novel Fiber Optical Strain Sensor for Monitoring the Vertical Deflection of an Aircraft Flap. IEEE Sensors Journal, 910), 1219-1225. doi:10.1109/jsen.2009.2029109

[6]Guyader, G., Gabor, A., \& Hamelin, P. (2013). Analysis of 2D and 3D circular braiding processes: Modeling the interaction between the process parameters and the pre-form architecture. Mechanism and Machine Theory, 69, 90-104. doi:10.1016/j.mechmachtheory.2013.04.015

[7]Jiang, N., \& Hu, H. (2019). Auxetic Yarn Made with Circular Braiding Technology. physica status solidi (b), 256(1), 1800168. doi:10.1002/pssb.201800168

[8]Kim, D.-H., \& Feng, M. Q. (2007). Real-Time Structural Health Monitoring Using a Novel FiberOptic Accelerometer System. IEEE Sensors Journal, 7(4), 536-543. doi:10.1109/jsen.2007.891988

[9]Kim, H.-Y., Lee, J.-H., \& Kim, D.-H. (2014). Muscular Condition Monitoring System Using Fiber Bragg Grating Sensors. Journal of the Korean Society for Nondestructive Testing, 34(5), 362-368. doi:10.7779/jksnt.2014.34.5.362

[10]Koerdt, M., Kibben, S., Hesselbach, J., Brauner, C., Herrmann, A. S., Vollertsen, F., \& Kroll, L. (2014). Fabrication and Characterization of Bragg Gratings in a Graded-index Perfluorinated Polymer Optical Fiber. Procedia Technology, 15, 138-146. doi:10.1016/j.protcy.2014.09.065

[11]Kong, Y., Abdullah, S., Schramm, D., Omar, M., \& Haris, S. (2018). Vibration Fatigue Analysis of Carbon Steel Coil Spring under Various Road Excitations. Metals, 8, 617. doi:10.3390/met8080617

[12]Lacraz, A., Polis, M., Theodosiou, A., Koutsides, C., \& Kalli, K. (2015). Femtosecond Laser Inscribed Bragg Gratings in Low Loss CYTOP Polymer Optical Fiber. IEEE Photonics Technology Letters, 27(7), 693-696. doi:10.1109/lpt.2014.2386692

[13]Marquez-Cruz, V., \& Albert, J. (2015). High Resolution NIR TFBG-Assisted Biochemical Sensors. Journal of Lightwave Technology, 33(16), 3363-3373. doi:10.1109/jlt.2015.2431912 
[14]Rawal, A., Gupta, S., Saraswat, H., \& Sibal, A. (2015). Geometrical modeling of near-net shape braided preforms. Textile Research Journal, 85(10), 1055-1064. doi:10.1177/0040517514559587

[15]Schwartz, M., \& Dixon, P. C. (2018). The effect of subject measurement error on joint kinematics in the conventional gait model: Insights from the open-source pyCGM tool using high performance computing methods. PLoS One, 13(1), e0189984. doi:10.1371/journal.pone.0189984

[16]SHANG, Z. U. F. E. N. G., WANG, S. H. U. X. I. N., YOU, Z. H. O. N. G., \& MA, J. I. A. Y. A. O. (2019). A HYBRID TUBULAR BRAID WITH IMPROVED LONGITUDINAL STIFFNESS FOR MEDICAL CATHETER. Journal of Mechanics in Medicine and Biology, 1903), 1950003. doi:10.1142/s0219519419500039

[17]Soh, D. B. S., Nilsson, J., Sahu, J. K., \& Cooper, L. J. (2003). Geometrical factor modification of helical-core fiber radiation loss formula. Optics Communications, 222(1-6), 235-242. doi:10.1016/s0030-4018(03)01599-2

[18]Sry, V., Mizutani, Y., Endo, G., Suzuki, Y., \& Todoroki, A. (2018). Estimation of the Longitudinal Elasticity Modulus of Braided Synthetic Fiber Rope Utilizing Classical Laminate Theory with the Unit N/tex. Applied Sciences, 8(7), 1096. doi:10.3390/app8071096

[19]Subramani, P., Rana, S., Ghiassi, B., Fangueiro, R., Oliveira, D. V., Lourenco, P. B., \& Xavier, J. (2016). Development and characterization of novel auxetic structures based on re-entrant hexagon design produced from braided composites. Composites Part B: Engineering, 93, 132-142. doi:10.1016/j.compositesb.2016.02.058

[20]Sun, A. (2012). Hybrid long-period-grating and fiber Bragg grating for cladding-mode-recoupling-based discrimination of temperature and strain. Optical Engineering, 51(4), 044402. doi:10.1117/1.oe.51.4.044402

[21]Tiwari, U., Mishra, V., Bhalla, A., Singh, N., Jain, S. C., Garg, H., ... Kapur, P. (2011). Fiber Bragg grating sensor for measurement of impact absorption capability of mouthguards. Dental Traumatology, 27(4), 263-268. doi:10.1111/j.1600-9657.2011.00998.x

[22]Udd, E. (2007). Review of multi-parameter fiber grating sensors. Proceedings from Fiber Optic Sensors and Applications $V$.

[23]Yan, Z., Sun, Q., Wang, C., Sun, Z., Mou, C., Zhou, K., ... Zhang, L. (2017). Refractive index and temperature sensitivity characterization of excessively tilted fiber grating. Opt Express, 25(4), 33363346. doi:10.1364/OE.25.003336

[24]Zhang, Y., Wang, F., Liu, Z., Duan, Z., Cui, W., Han, J., . . Peng, W. (2017). Fiber-optic anemometer based on single-walled carbon nanotube coated tilted fiber Bragg grating. Optics Express, 25(20), 24521. doi:10.1364/oe.25.024521

[25]Zhang, Y., Meng, Z., Hu, X., Su, L., \& Sun, Y. (2018). Modeling and analysis of friction in endface/inner-face circular braiding processes. The Journal of The Textile Institute, 109(11), 1400-1408. doi:10.1080/00405000.2018.1423900

[26] Zhifeng, Z. (2015). Polymer Optical Fiber Bragg Grating. In Handbook of Smart Textiles (pp. 597613). Singapore: Springer Singapore. doi:10.1007/978-981-4451-45-1_27

[27]Zhou, W., Mandia, D. J., Barry, S. T., \& Albert, J. (2015). Absolute near-infrared refractometry with a calibrated tilted fiber Bragg grating. Opt Lett, 4O(8), 1713-1716. doi:10.1364/OL.40.001713 